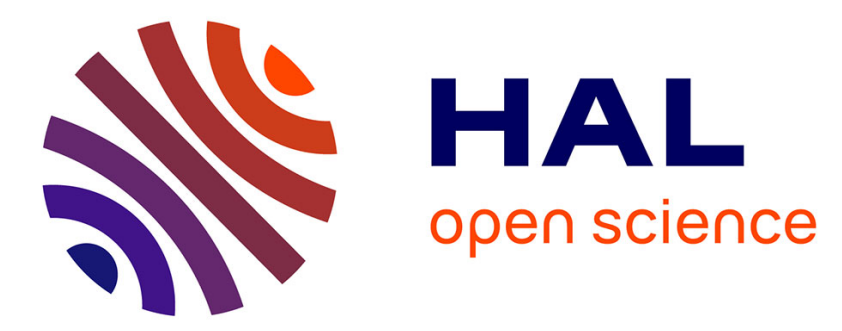

\title{
The astrometry of the natural planetary satellites applied to their dynamics before and after Gaia
} Jean-Eudes Arlot, Josselin Desmars, Valéry Lainey, Vincent Robert

\section{To cite this version:}

Jean-Eudes Arlot, Josselin Desmars, Valéry Lainey, Vincent Robert. The astrometry of the natural planetary satellites applied to their dynamics before and after Gaia. Planetary and Space Science, 2012, 73 (1), pp.66-69. 10.1016/j.pss.2012.10.002 . hal-02944919

\section{HAL Id: hal-02944919 \\ https://hal.science/hal-02944919}

Submitted on 21 Sep 2020

HAL is a multi-disciplinary open access archive for the deposit and dissemination of scientific research documents, whether they are published or not. The documents may come from teaching and research institutions in France or abroad, or from public or private research centers.
L'archive ouverte pluridisciplinaire HAL, est destinée au dépôt et à la diffusion de documents scientifiques de niveau recherche, publiés ou non, émanant des établissements d'enseignement et de recherche français ou étrangers, des laboratoires publics ou privés. 


\title{
The astrometry of the natural planetary satellites applied to their dynamics before and after Gaia
}

\author{
Jean-Eudes Arlot*, Josselin Desmars, Valéry Lainey, Vincent Robert \\ Paris Observatory/IMCCE/USTL/LASA/IPSA/UPMC/CNRS, 77 Avenue Denfert-Rochereau, F-75014 Paris, France
}

\section{A R T I C L E I N F O}

\section{Article history:}

Received 23 November 2011

Received in revised form

4 September 2012

Accepted 5 October 2012

Available online 17 October 2012

\section{Keywords:}

Astrometry

GAIA

Natural satellites

Celestial mechanics

\begin{abstract}
A B S T R A C T
The natural planetary satellites are observed since centuries in order to understand their dynamics and their evolution. A high level astrometric accuracy is necessary for this purpose. The arrival of Gaia makes necessary to perform the ground-based observations in the scope of the future data provided by Gaia. About 50 observations by Gaia of each satellite will be available, and the Gaia star catalogue will allow to reduce the astrometric observations with a high level accuracy. This is true for observations made at the present time, made in the future, and made in the past thanks to a new reduction of old observations such as photographic plates. We analyse the different status of the satellites depending on the system of which they belong. This will decide the astrometric observing programmes of the natural planetary satellites for the next years.
\end{abstract}

(c) 2012 Elsevier Ltd. All rights reserved.

\section{Introduction}

Astrometric observations of the natural planetary satellites are regularly performed. The frequency of the observations must depend on the satellites, on their revolution period around their planet, and on the nature of the gravitational perturbations. The arrival of Gaia leads us to consider new plans for the observing programmes and the reduction of the observations.

Sections 2 and 3 present the astrometry of the natural planetary satellites and the status of the present astrometric reduction. Sections 4 and 5 explain how Gaia will modify the astrometry of the natural satellites and Section 6 what we have to do for preparing the arrival of Gaia observations.

\section{The astrometry of the natural planetary satellites}

(1) Purpose. The astrometry of the natural planetary satellites has several goals. The knowledge of their dynamics helps us to understand the formation and evolution of the solar system. Moreover the internal structure of the natural satellites may be understood through the dynamics; tidal effects and dissipation induce an acceleration in their motions which is detectable through the astrometric observations (Lainey et al., 2009).

We have to note also that the astrometry of the giant planets is possible only through their satellites (natural or artificial,

\footnotetext{
* Corresponding author.

E-mail addresses: arlot@imcce.fr, jean-eudes.arlot@imcce.fr (J.-E. Arlot).
}

indirectly with space probes). Only the satellites are observable accurately and the position and motion of the planets are deduced from the ephemerides of the satellites (Robert et al., 2011). For these purposes, we need accurate astrometric observations of the satellites to build accurate dynamical models. From the positions of the satellites, we may derive the position of the planet by translating the observed positions of the satellites to observed positions of the center of mass of the primary.

In both cases, we need observations spread over a long interval of time depending on the velocity of the satellites in order to model completely the dynamics of the satellites. How will Gaia help? Is the Gaia improvement the same for all satellites?

(2) The needed accuracy of the observations. An astrometric observation is valuable only if its accuracy is sufficient to assess significant dynamical effects.

For example, for the Galilean satellites of Jupiter, the tidal effects may infer forces (Lainey et al., 2009) which lead to a cumulative secular shift in longitude of about $100 \mathrm{~m}$ by year. On this basis, Table 1 provides the observing time interval necessary to detect this effect in the observations depending of their accuracy (with a signal/noise ratio of 2). Of course some selected sets of observations may have a better accuracy but not on the whole needed interval of time (Lainey et al., 2004). Note that another constraint has to be taken into an account: the long period terms which may be separated from the secular dissipative effects. For that, a minimum of 50 yrs of observations is necessary independently from the accuracy of the observations.

Of course, we need a large number of observations spread on a long interval of time and, due to the non-uniform distribution of observation with time and due to unexpected systematic errors 
depending on the observing techniques, all types of data are useful and may be mixed.

(3) Sampling the observations. The Gaia observations will be done on an interval of time limited to 5 yrs. Is this duration sufficient? In order to know the necessary length of this interval of time, we simulated observations and built ephemerides. Then, it appeared that one century of ground-based observations is equivalent to $5 \mathrm{yrs}$ of Gaia observations (Desmars, 2009a; Desmars et al., 2009b). The accuracy of the dynamical models does not depend only on the accuracy of the observations but also on the length of the period of observations. This may be tested through the extrapolation of the ephemerides depending on the sampling of observations used to fit the theoretical model of motion. For example, for Mimas, we split the set of available observations (Desmars et al., 2009c) into two sets: the first one gathering 1547 observations made on a 73 yrs interval of time

Table 1

Intervals of observing time depending on the type of observations.

\begin{tabular}{lcc}
\hline Observations & Accuracy $(\mathrm{km})$ & Observing interval $(\mathrm{yrs})$ \\
\hline Jovian eclipses & 500 & 100 \\
Old photographic & 300 & 75 \\
CCD images & 150 & 50 \\
Mutual events & 50 & 30 \\
Space probes & 5 & 10 \\
\hline
\end{tabular}
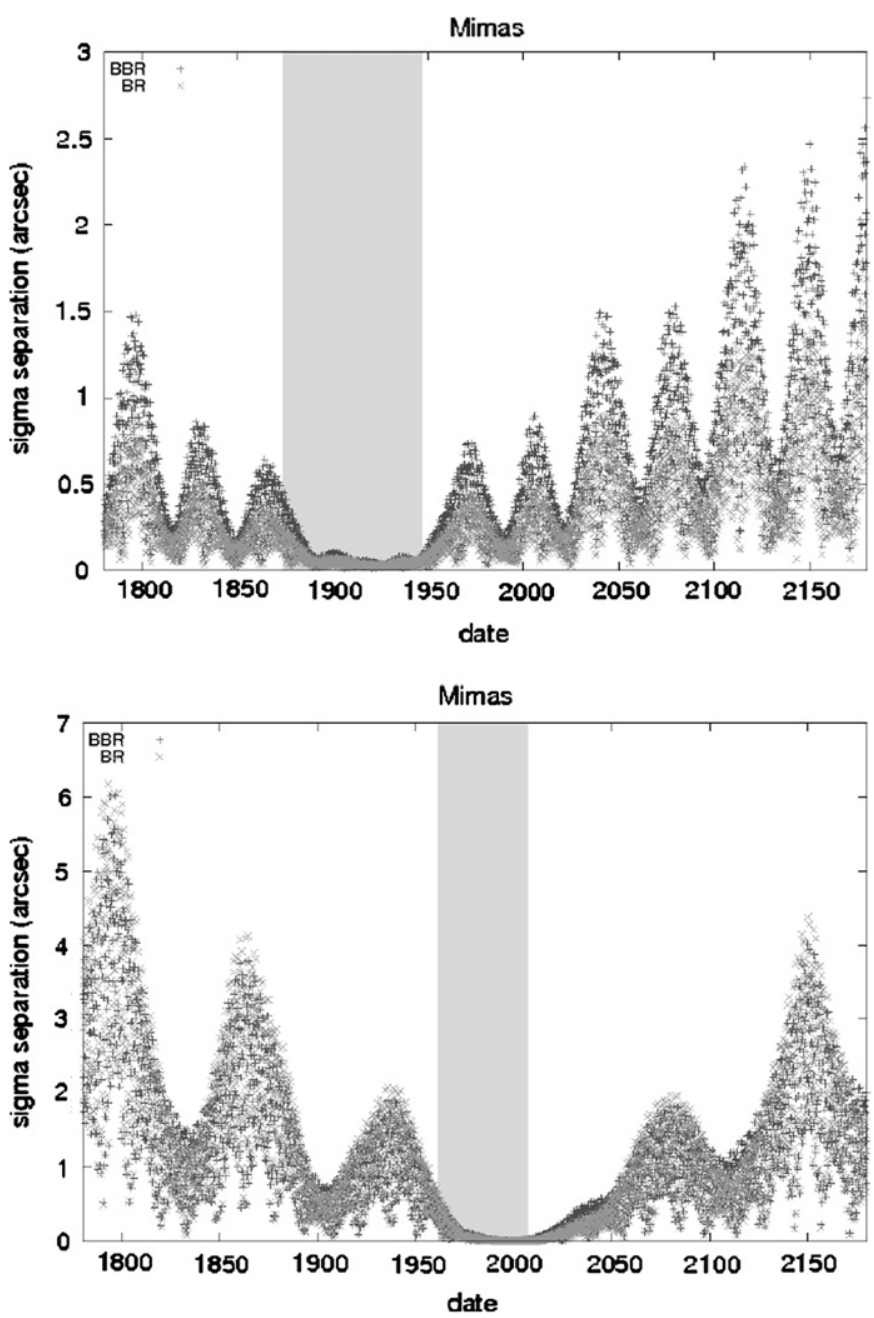

Fig. 1. Accuracy of extrapolated ephemerides depending on the observations used for the fit.
(1874-1947), the accuracy of which being 0.30 arcsec and the second one gathering 2820 observations made on a 46 yrs interval of time (1961-2007) the accuracy of which being 0.15 arcsec. We then built ephemerides using each set, and extrapolated each ephemerides on the period of the other set of observations. We were able to see which ephemerides were the closer to the observations. One would expect that the large number of more accurate observations will provide the smallest error for the ephemerides but in fact the contrary occurs. As seen on Fig. 1, the ephemerides made with the old less accurate observations are better than the other one. This comes from a periodic term of $70 \mathrm{yrs}$ in the motion of Mimas which is better quantified by observations spanning on 50 yrs than on 30 yrs.

Then the question of the use of the observations of the natural satellites by Gaia is appropriate.

\section{The astrometric reduction before Gaia: accuracy of catalogues}

Astrometric reduction is performed by linking the catalogued stars with field stars. Then, the accuracy will depend on the field recorded together with the studied object. Small fields have less distorsion due to optics but they contain less catalogued stars. For example, a good catalogue published in 1997 after the astrometric satellite Hipparcos contains only 120,000 stars until magnitude 12.4 with an accuracy better than 1 mas. The Tycho catalogue deduced from the Hipparcos mission contains 2,500,000 stars until magnitude 16 but with an accuracy not better than 60 mas due to proper motions. Small fields needing fainter stars, some catalogues intent to compile a larger number of stars: the USNO SA2 contains $526,000,000$ stars with an accuracy not better than 150 mas. It is preferable, when the field is not too small to use the UCAC2 catalogue containing 48,330,571 stars with magnitude from 7.5 to 16 with an accuracy around 50 mas. One will understand easily that the Gaia catalogue will replace all the older catalogues for astrometric reduction.

\section{The satellites observed by Gaia: the possible accuracy}

We know that Gaia will observe all natural satellites the magnitude of which being from 8 to 20 with an accuracy around 1 mas. Each object will be observed about 50 times during a 5 yrs period, a little less for satellites close to their planet due to possible occultation or blooming due to the bright primary. The accuracy and the interest of these observations will depend on the object:

- Large objects extensively observed from Earth since years and observed by space probes will not take a large benefit from Gaia direct observations.

- Objects close to the primary, difficult to observe from groundbased observatories, will take benefit from Gaia direct observations, events the vicinity of the planet will allow Gaia to observe less than 50 times as stated above.

- Small outer satellites far from their planet are similar to asteroids. Gaia observations will be valuable providing accurate positions, photometry, and shapes of the objects. Anyway, this final astrometric accuracy will be around 1-2 mas that will be a real improvement.

Table 2 provides an estimation of the interest of the Gaia direct observations depending on the satellites. 
Table 2

Usefulness of the Gaia data depending on satellites: + means usefulness.

\begin{tabular}{|c|c|c|c|c|}
\hline Satellites & Period & Gaia observations & Observations & Gaia catalogue \\
\hline Close to the planet & Hours & ++ & Difficult ground-based observations & + \\
\hline Large observed by Gaia & Days & - & Obs. on long time interval & ++ \\
\hline Large not observed by Gaia & Days & No & Improvement of ground-based observations & $+11++$ \\
\hline Outer irregular mag $<20$ & Years & $+1+1+$ & Getting shape and precise observations by Gaia & ++ \\
\hline Outer irregular mag $>20$ & Years & No & Improvement of ground-based observations & $+11++$ \\
\hline
\end{tabular}

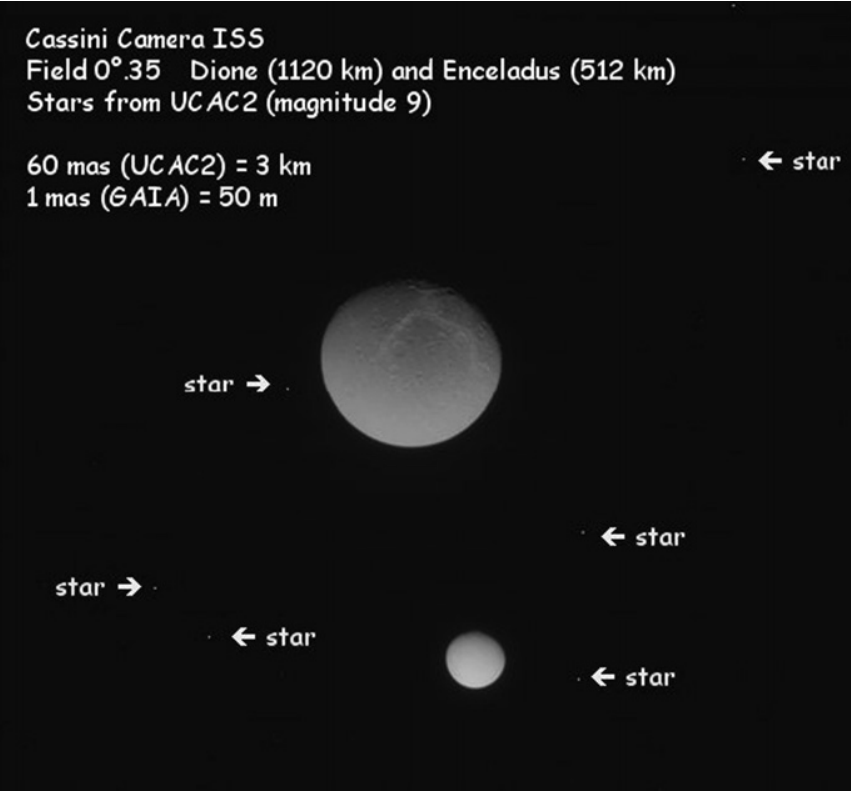

Fig. 2. Dione and Enceladus observed by the Cassini space probe: the astrometric reduction uses the stars present in the field.

\section{Future astrometric reduction using the Gaia star catalogue}

Future observations will use the Gaia catalogue for the astrometric reduction. The accuracy of the astrometric observations will not depend on the Gaia star catalogue but on the telescope used, the receptor, the measuring algorithm, the signal/noise ratio, the size of the object, the knowledge of its surface and shape. The Gaia catalogue will contain 1 billion stars until magnitude 20, so even a small field (a few arcmin) will contain a sufficient number of stars for the reduction.

Old observations will also take benefit from the Gaia astrometric star catalogue. A new reduction of old observations has to be considered and old observations have to be chosen carefully, depending of their quality and of the scientific need.

Fig. 2 provides an image taken by the Cassini space probe, showing Enceladus and Dione against a field of a few stars. These stars are used for the astrometric reduction and allow to obtain positions of the two satellites with an accuracy of $5 \mathrm{~km}$, mainly due to the uncertainty of the star catalogue. When doing the reduction with the Gaia catalogue, the uncertainty would decrease to $100 \mathrm{~m}$, but the difficulty to measure the positions of the satellites will increase the uncertainty up to 1 or $2 \mathrm{~km}$. However, this improvement justifies a new reduction.

Fig. 3 provides an image of the scan of the central part of a photographic plate taken in 1974 at USNO and showing several exposures of the Galilean satellites of Jupiter. These satellites are difficult to observe because they are too bright (which is the reason why Gaia will not observe them). The plates include a few stars which were not catalogued at the time of the observation. Recently, these

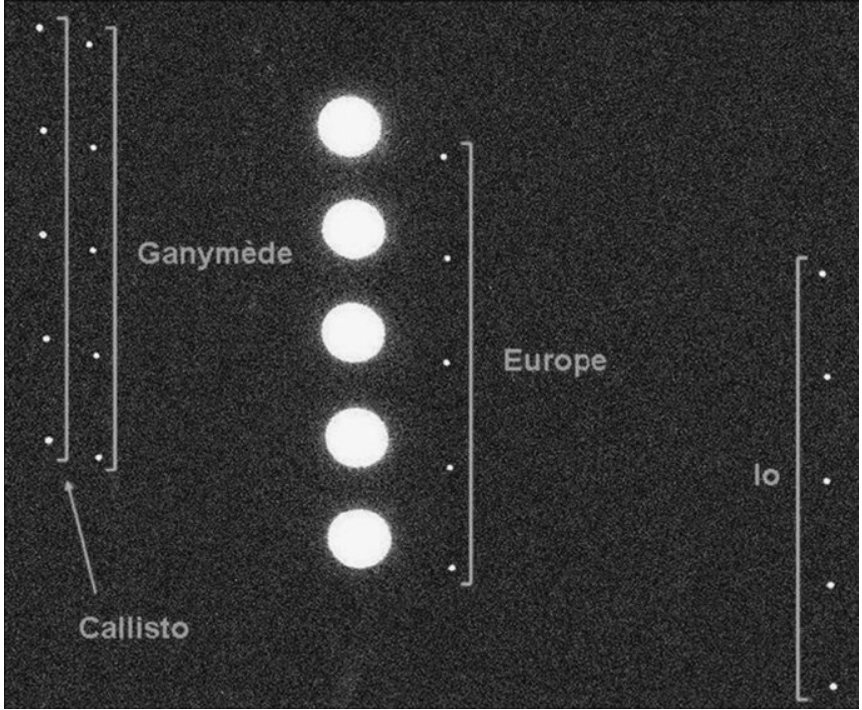

Fig. 3. The center of a photographic plate of the Galilean satellites made at USNO in 1974 .

plates were scanned and reduced with the UCAC2 catalogue providing very accurate and interesting results for the satellites and for the planet Jupiter (Robert et al., 2011). Making a new reduction with the Gaia catalogue will increase again the astrometric accuracy allowing to reach small effects in the orbital motion that is needed and provides interesting results (Lainey et al., 2009). Fig. 4 shows the present accuracies of the observations of the Galilean satellites. All the old photographic observations will have the accuracy of the measurement on the receptor after a new reduction with the Gaia catalogue, i.e. an accuracy between 2 and 10 mas.

\section{Future work}

After the arrival of Gaia data, we will first use the 50 GAIA observations and second, we will use the Gaia star catalogue for the reduction of the new CCD astrometric observations. Then, we will re-reduce old observations: a long work of searching and selecting old observations among several hundreds of thousands of plates spread in observatories worldwide. Metadata should be available to perform a new reduction. All of this will allow a better dynamical modeling of these solar system objects, a better measurement of tidal effects and a better understanding of the evolution of the satellites systems. Table 2 summarises the interest of Gaia direct observations and Gaia star catalogue depending on the satellites.

\section{Conclusion}

The usefulness of the Gaia data for the natural satellites study will depend on the satellites. We have to be prepared for the 


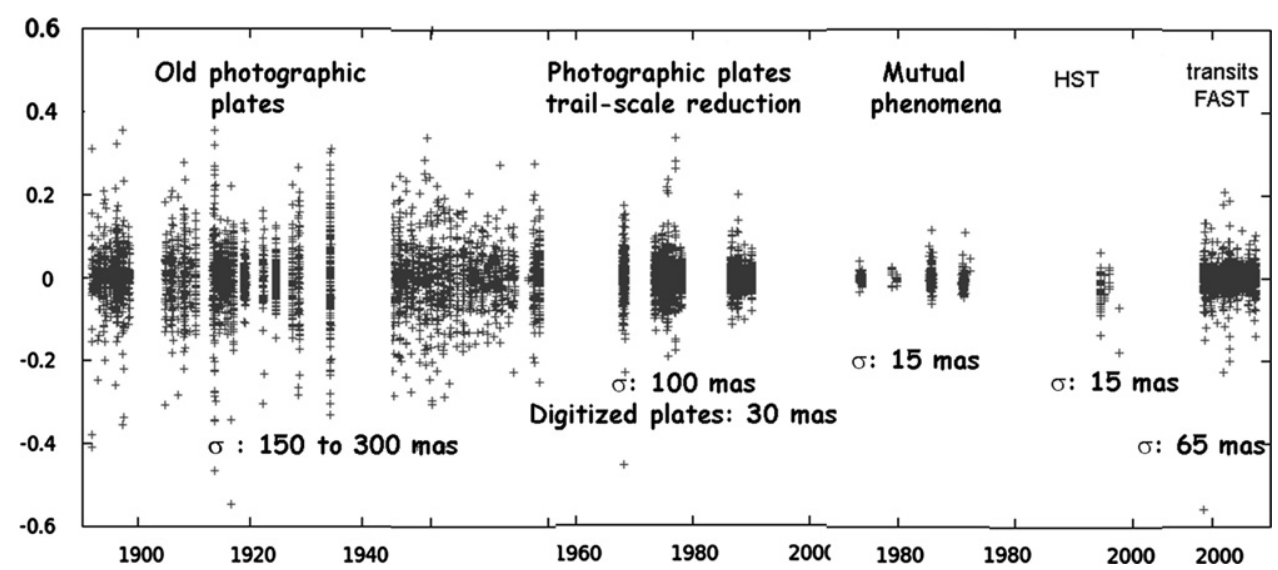

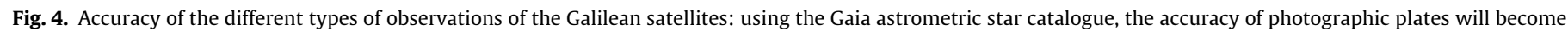
around 10 mas. Note that 1 mas corresponds to $3 \mathrm{~km}$.

arrival of Gaia data and the observations made at the present must take this fact into an account. Because of the high velocity of most of the natural planetary satellites, we must never stop observing even if you have to wait for the release of the Gaia catalogue for an accurate reduction; an observation not made is a missing observation. Good observational sampling is crucial. More, we should decide as soon as possible what old data should be re-reduced with the Gaia astrometric star catalogue. Due to the high accuracy of positions and proper motions, the Gaia astrometric catalogue will be good enough even for old observations made one century ago, when the photographic plates appeared. Then a re-reduction of old plates with the Gaia star catalogue will provide new valuable information to aid the study of natural satellites.

\section{Acknowledgements}

Part of this work was supported by the UPMC Contract EME0911. We are grateful to Radwan Tajeddine for providing us images from the Cassini space probe.

\section{References}

Desmars, J., 2009a. Precision d'extrapolation des ephemerides dans le systeme solaire. Ph.D. Paris Observatory, Paris.

Desmars, J., Arlot, J.-E., Arlot, S., Lainey, V., Vienne, A., 2009b. Estimating the accuracy of satellites ephemerides using the bootstrap method. Astronomy and Astrophysics 499, 321.

Desmars, J., Vienne, A., Arlot, 2009c. A new catalogue of observations of the eight major satellites of Saturn (1874-2007). Astronomy and Astrophysics 493, 1183.

Lainey, V., Arlot, J.E., Karatekin, O., Van Hoolst, T., 2009. Strong tidal dissipation in Io and Jupiter from astrometric observations. Nature 459, 957.

Lainey, V., Arlot, J.E., Vienne, A., 2004. New accurate ephemerides for the Galilean satellites of Jupiter. II. Fitting the observations. Astronomy and Astrophysics 427, 371.

Robert, V., De Cuyper, J.P., Arlot, J.E., de Decker, G., Guibert, J., Lainey, V., Pascu, D., Winter, L., Zacharias, N., 2011. A new astrometric reduction of photographic plates using the DAMIAN digitizer: improving the dynamics of the Jovian system. Monthly Notices of the Royal Astronomical Society 415, 701. 